\title{
Thermal Transport through Single-Molecule Junctions
}

\author{
Nico Mosso, ${ }^{\dagger}$ Hatef Sadeghi, ${ }^{*}, \S \odot$ Andrea Gemma, ${ }^{\dagger}$ Sara Sangtarash, ${ }^{\ddagger}, \S$ Ute Drechsler, ${ }^{\dagger}$ \\ Colin Lambert, ${ }^{\S}$ and Bernd Gotsmann ${ }^{*}, \dagger$ (1)
}

${ }^{\dagger}$ IBM Research-Zurich, Rueschlikon 8803, Switzerland

${ }^{\ddagger}$ School of Engineering, University of Warwick, Coventry CV4 7AL, United Kingdom

${ }^{\S}$ Physics Department, Lancaster University, Lancaster LA1 4YB, United Kingdom

\section{Supporting Information}
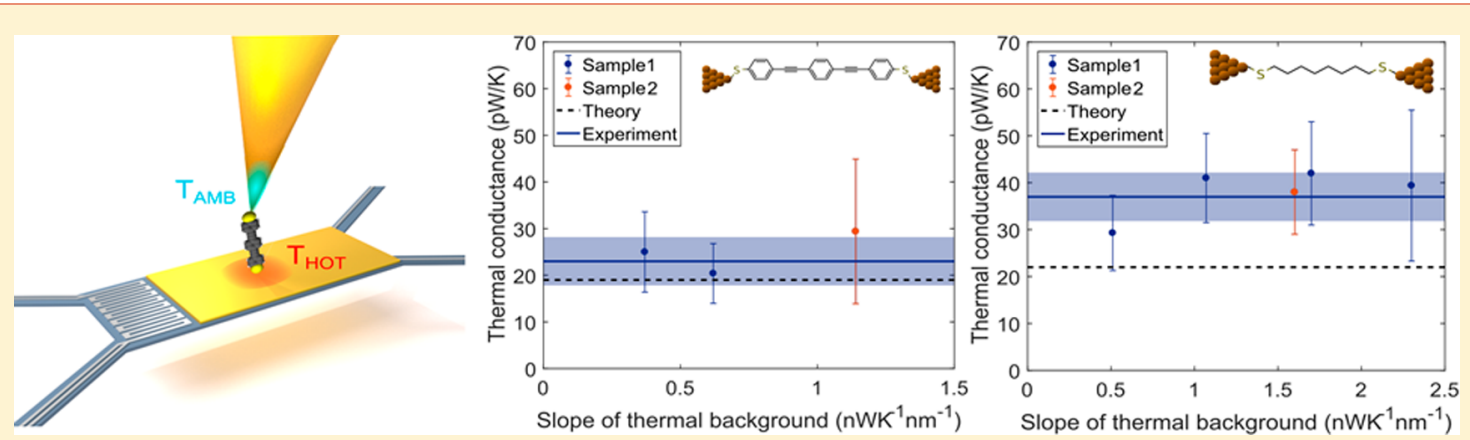

ABSTRACT: Molecular junctions exhibit a rich and tunable set of thermal transport phenomena. However, the predicted high thermoelectric efficiencies, phonon quantum interference effects, rectification, and nonlinear heat transport properties of organic molecules are yet to be verified because suitable experimental techniques have been missing. Here, by combining the break junction technique with suspended heat-flux sensors with picowatt per Kelvin sensitivity, we measured the thermal and electrical conductance of single organic molecules at room temperature simultaneously. We used this method to study the thermal transport properties of two model systems, namely, dithiol-oligo(phenylene ethynylene) and octane dithiol junctions with gold electrodes. In agreement with our density functional theory and phase-coherent transport calculations, we show that heat transport across these systems is governed by the phonon mismatch between the molecules and the metallic electrodes. This work represents the first measurement of thermal transport through single molecules and opens new opportunities for studying heat management at the nanoscale level.

KEYWORDS: Thermal transport, single molecule, molecular junctions

$\mathrm{H}$ eat transport through molecular systems takes place through a wealth of transport mechanisms. When the heat is carried by vibrations, these include ballistic and hopping transport, phonon interference, ${ }^{1-3}$ rectification $^{4}$ and localization, ${ }^{5}$ and extend beyond equilibrium-based thermodynamic transport. ${ }^{6}$ Molecular junctions represent an ideal platform to probe quantum transport phenomena at the nanoscale, and investigation of charge transport in these systems has led to numerous fundamental discoveries in the last 20 years. ${ }^{7}$ Tuning the thermal properties of such molecular devices would underpin numerous technologies based on heat management at the nanoscale. However, the thermal characterization of molecular junctions has not been experimentally accessible yet.

Both experimental and theoretical analyses of molecular heat transport face severe challenges. To simulate phononic heat transport in molecular junctions, different length scales of heatcarrying phonons have to be taken into account, ranging from Ångstroms to microns at ambient temperature. Molecular dynamics simulations have been utilized in the classical or high-temperature limit to identify the role of binding groups and interference effects. ${ }^{1}$ More recently, significant progress was made describing junctions as Landauer systems with an energy-dependent phonon transmission coefficient calculated using Green's functions and density functional theory, ${ }^{2,5,8}$ or approaches beyond the harmonic approximation. ${ }^{6}$ The Green's function method has also been employed to compute the electronic contribution to heat transport. However, numerous predictions of interference phenomena ${ }^{2,9,10}$ and nonlinear effects ${ }^{4}$ await experimental verification.

The thermal conductance of a single molecular junction is typically well below a thermal conductance quantum (284 $\mathrm{pW} / \mathrm{K}$ at room temperature) and therefore difficult to measure. For example, alkanes chemically bound to two metal thermal reservoirs, have measured and predicted thermal conductance values on the order of tens of picowatts per Kelvin. ${ }^{6,11}$ As these systems form self-assembled monolayers of high quality, they can be measured using spatially averaging methods like time/frequency-domain thermoreflectance ${ }^{12,13}$ or

Received: May 22, 2019

Revised: September 17, 2019

Published: September 27, 2019 

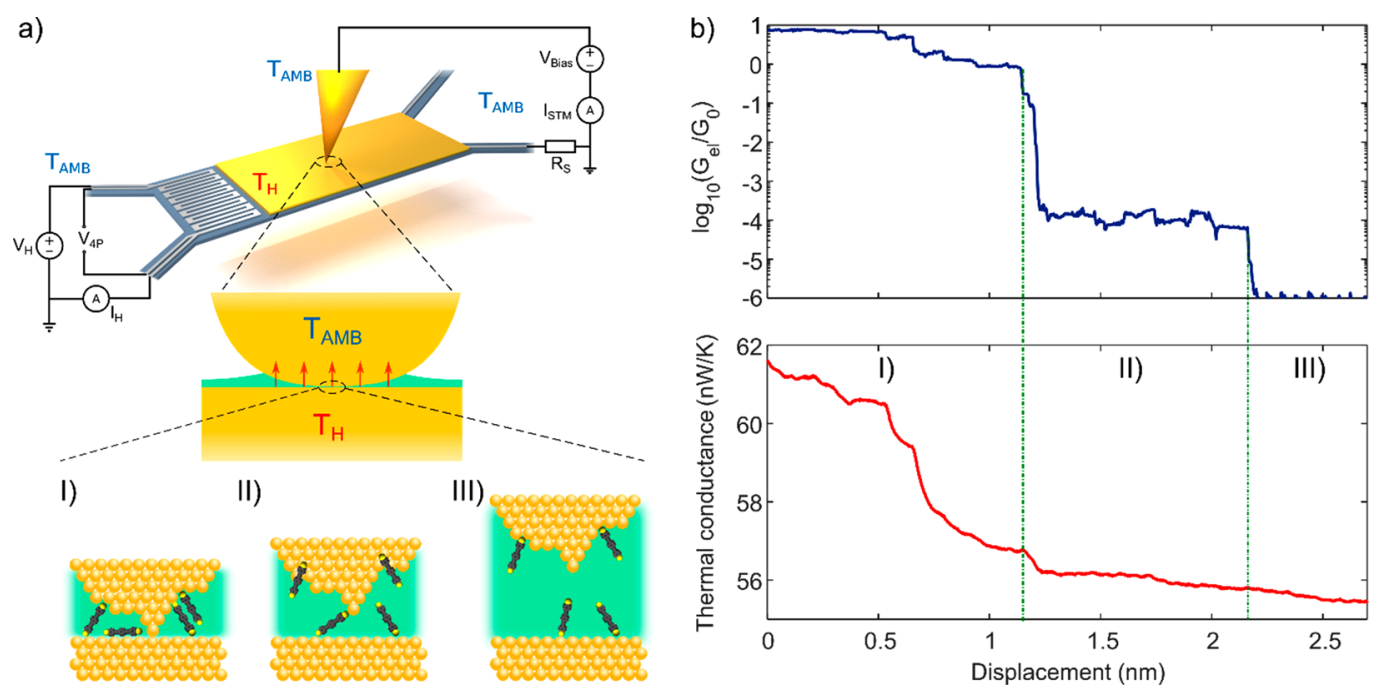

Figure 1. Schematic of the measurement technique. (a) Schematic diagram of the experiment with the different transport regimes I-II-III upon breaking the tip-MEMS contact. The green meniscus represents the thermal contact with adsorbates on the MEMS. The thermal conductance of the junction is obtained by measuring the four-probe resistance of the platinum heater $\left(R=V_{4 \mathrm{P}} / I_{\mathrm{H}}\right)$, which is directly related to its temperature $T_{\mathrm{H}}$, and the total electrical power dissipated. Simultaneously, the electrical conductance is calculated from the tunneling current, $I_{\text {STM }}$, measured at a fixed bias voltage, $V_{\text {bias. }}$ (b) Example of a typical opening trace showing the variation of the electrical conductance of the junction $G_{\mathrm{el}}$ normalized by the conductance quantum $G_{0}=2 \mathrm{e}^{2} / h$ and the overall thermal conductance of the Tip-MEMS system versus the tip displacement. (I) Au-Au contact. (II) Formation of a single-molecule junction indicated by the electrical conductance plateau. The variation in thermal conductance can be due to a modulation of the contact area with the adsorbates. (III) Breaking of the molecular junction, resulting in a small decrease of the overall thermal conductance, on the scale of a few tens of $\mathrm{pW} / \mathrm{K}$.

scanning thermal microscopy. ${ }^{14}$ However, for most molecular systems, ordered films cannot readily be made and uncertainties in the actual percentage of molecules bridging the reservoirs still remain. ${ }^{13,14}$

Even though single-molecule measurements are prone to difficulties, due to the experimental inaccessibility of the atomic-scale environment of an individual molecular junction, they would enable systematic studies of the relationship between chemical structure and energy transport properties. Indeed recently, experiments using break-junction techniques have been used to probe the thermal conductance of atomic point contacts. $^{15,16}$

Here, for the first time, we demonstrate how a combination of a break junction with a suspended microfabricated heat flux sensor can be used to probe the thermal conductance of molecular junctions. As model molecular systems, we have chosen oligo(phenylene ethynylene)dithiol (OPE3) and octaneditiol (ODT) contacted by gold electrodes. OPE3 is probably the best-studied molecule for electrical transport, with a widespread agreement reached by different groups for its electrical conductance, ${ }^{17-20}$ using both mechanically controlled break-junctions $^{2}$ (MCBJ) and break junctions based on scanning tunneling microscopes (STM-BJ). However, heat transport through OPE3 with thiolate end groups has neither been studied experimentally nor theoretically. On the other hand, ODT is a member of the alkane family, whose thermal transport properties have been intensively studied both theoretically and experimentally. Nevertheless, the many possible junction configurations recognized in the charge transport experiments, ${ }^{21-24}$ make it a challenging system to test the limits of our new method.

The experimental setup is represented schematically in Figure 1a. In order to combine heat and charge transport measurements at the single-molecule level, we devised suspended micro-electro-mechanical systems (MEMS) $)^{15}$ char- acterized by a low thermal conductance $G_{\text {MEMS }}$ ranging from 3.5 to $4.5 \times 10^{-8} \mathrm{~W} / \mathrm{K}$. These devices consist of a central membrane with a Pt heater/thermometer and a gold platform that can be used to form electrical contacts with the tip of a scanning tunneling microscope (STM). The membrane is suspended via four silicon nitride beams, which are usually oriented at $45^{\circ}$ with respect to its axis to reduce the torsional degrees of freedom. The MEMS are cleaned by a combination of oxygen plasma and ion milling to remove contaminants postfabrication and retrieve a fresh gold surface. OPE3 or ODT are deposited on the gold platform by immersing the entire MEMS in solutions of dichloromethane or ethanol, respectively, with concentrations ranging from 0.1 to $1 \mathrm{mM}$ for $30 \mathrm{~s}$ to $2 \mathrm{~h}$. After the deposition, the samples are rinsed several times in clean solvent to eliminate physically adsorbed molecules. For a detailed description of the sample preparation procedure, see the Supporting Information. We would like to stress that depositing molecules from solution poses severe challenges on the cleanliness of the gold surface, but it extends considerably the variety of molecules that can be investigated.

All the measurements are performed at room temperature and in high vacuum $\left(\sim 10^{-7} \mathrm{mbar}\right)$ within a custom-built STM, located in a low-noise environment. ${ }^{25}$ The experiment consists in performing STM-Break Junction (STM-BJ) measurements with an electrochemically etched gold tip (typical tip radius around $50 \mathrm{~nm}$ ) on the gold platform of the suspended MEMS. Measuring the electrical conductance $G_{\mathrm{el}}$ upon breaking the tip-MEMS contact allows us to detect the formation of a molecular junction. Prior to contact formation, the membrane is heated to a temperature $T_{\mathrm{H}} \sim 350 \mathrm{~K}$ by applying a constant voltage to the Pt-heater, which corresponds to few $\mu \mathrm{W}$ of dissipated power. The temperature $T_{H}$ is continuously monitored by measuring the four-probe resistance of the heater and using the previously calibrated temperature coefficient of resistivity $\alpha=1.37 \times 10^{-3} \mathrm{~K}^{-1}$, which is in the 
a)

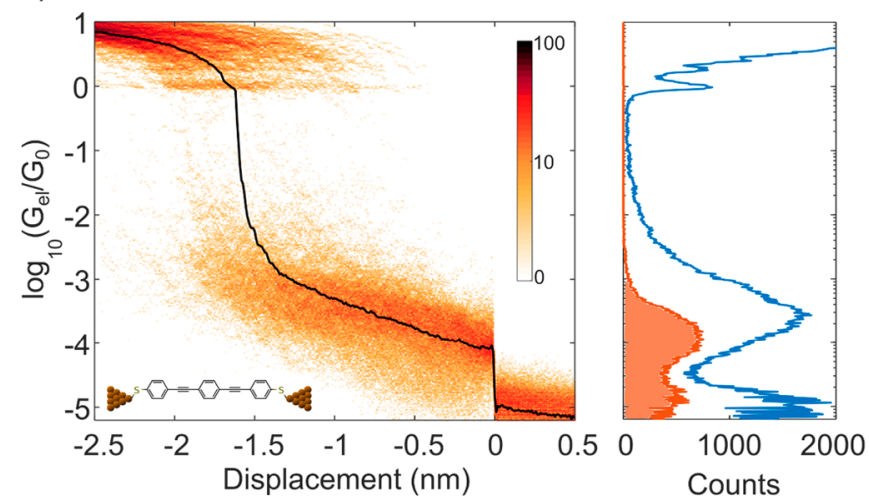

c)

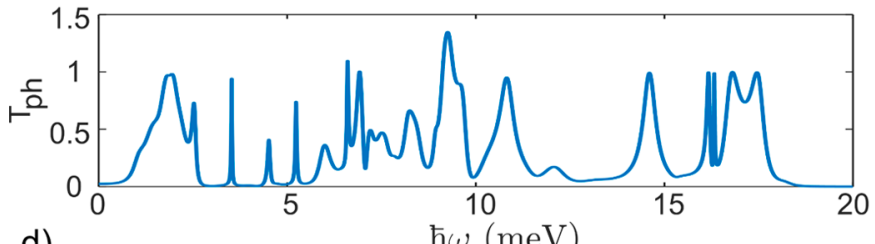

d)

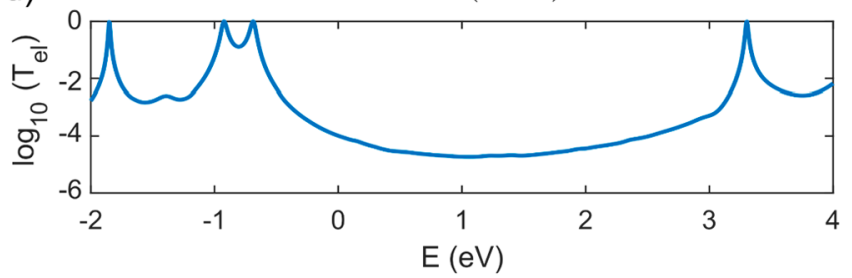

b)

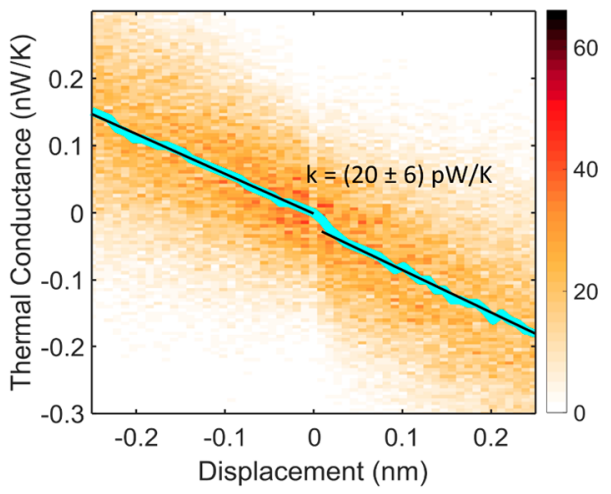

e)

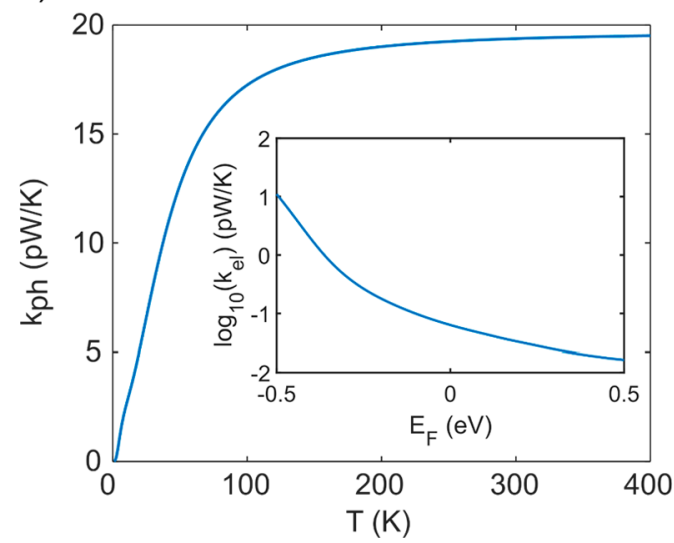

Figure 2. Experimental (a, b) and theoretical (c-e) results for OPE3-dithiol. (a) Electrical 2D and 1D histograms constructed with 411 traces measured at a fixed voltage of $50 \mathrm{mV}$ and a pulling speed $v=3 \mathrm{~nm} / \mathrm{s}$ at an angle of $40^{\circ}$ with respect to the MEMS surface. From the histograms, we can extract the molecular electrical conductance of about $2.7 \times 10^{-4} G_{0}$. The orange $1 \mathrm{D}$ histogram indicates the electrical conductance of the junction before breaking, reconstructed from the $2 \mathrm{D}$ histogram in the displacement range between -0.5 and $0.2 \mathrm{~nm}$. (b) $2 \mathrm{D}$ Thermal conductance histogram for OPE3. The width of the mean line (light blue) includes the total uncertainty of the extracted thermal conductance and shows a clear step around 0 (breaking point of the molecular junction). The black lines represent the linear fits of the mean before and after the step, giving a thermal conductance $k=(20 \pm 6) \mathrm{pW} / \mathrm{K}$. (c) Transmission coefficient $T_{\mathrm{ph}}$ of phonons with energy $\hbar \omega$. (d) Transmission coefficient $T_{\mathrm{el}}$ of electrons with energy $E$. (e) Calculated thermal conductance $k_{\mathrm{ph}}$ due to phonons versus temperature $T$. The inset shows the thermal conductance due to electrons versus the Fermi energy at room temperature.

expected range for Pt thin films. The thermal conductance $k_{\text {th }}$ of the MEMS is calculated by dividing the total power provided to the heater and the $\Delta T=T_{\mathrm{H}}-T_{\mathrm{AMB}}$ generated with respect to the substrate. When the tip, also at room temperature $T_{\mathrm{AMB}}$, is brought into contact with the MEMS, the total thermal conductance increases because of the additional heat flux to the tip. The difference between these two values corresponds to the thermal conductance of the contact formed.

Figure $1 \mathrm{~b}$ shows an example of a single opening trace measured by retracting the tip at $25^{\circ}$ with respect to the MEMS surface in order to take advantage of the greater inplane stiffness ${ }^{15}$ at a speed of $2 \mathrm{~nm} / \mathrm{s}$. Lower angles led to irreproducible results indicating that including roughness the local angle between tip and surface is smaller. We calculate the stiffness normal to the MEMS surface about $0.7 \mathrm{~N} / \mathrm{m}$ and the in-plane stiffness $540 \mathrm{~N} / \mathrm{m}$, resulting in an effective stiffness on the order of $10 \mathrm{~s}$ of $\mathrm{N} / \mathrm{m}$ (SI). Note that the plotted thermal conductance includes both the contributions of the MEMS $(\sim 45 \mathrm{nW} / \mathrm{K})$ and the contact with the tip. We can distinguish three different transport regimes within the trace: (I) $\mathrm{Au}-\mathrm{Au}$, (II) Au-molecule-Au, (III) Au-tunneling-Au. During the thinning of an $\mathrm{Au}-\mathrm{Au}$ point contact (I), the thermal conductance of the junction is proportional to the electrical one, decreasing in a step-like fashion because of the quantized availability of electron channels. Hereby, the last few channel changes coincide with the number of atoms bridging the contacts after rearrangement. Most of the heat is transported by electrons following the Wiedemann-Franz law. ${ }^{15,16}$ Some traces exhibit a second regime (II), in which $G_{\text {el }}$ shows a plateau indicating the formation of a molecular junction. ${ }^{26}$ Finally, in the last regime (III), the molecular junction is broken. The thermal conductance of the molecule can then be obtained from the signal change around the breaking point of the junction.

A similar plateau might be expected for the thermal conductance in the second regime (II). However, a linear decrease is usually observed versus tip displacement, with slopes ranging between 0.5 and $3 \mathrm{nW} /(\mathrm{K} \mathrm{nm})$. This is caused by additional thermal conductance paths around the actual molecular junction. These paths have relatively long-range and can be attributed to three effects. First, even clean gold surfaces can show significant heat transfer of this magnitude due to thermal near-field radiation. ${ }^{27,28}$ The magnitude of the nearfield contribution is under debate. ${ }^{27,28}$ Second, despite the cleaning processes described above, there may be residues and spurious molecules present on the surface from processing and 
a)

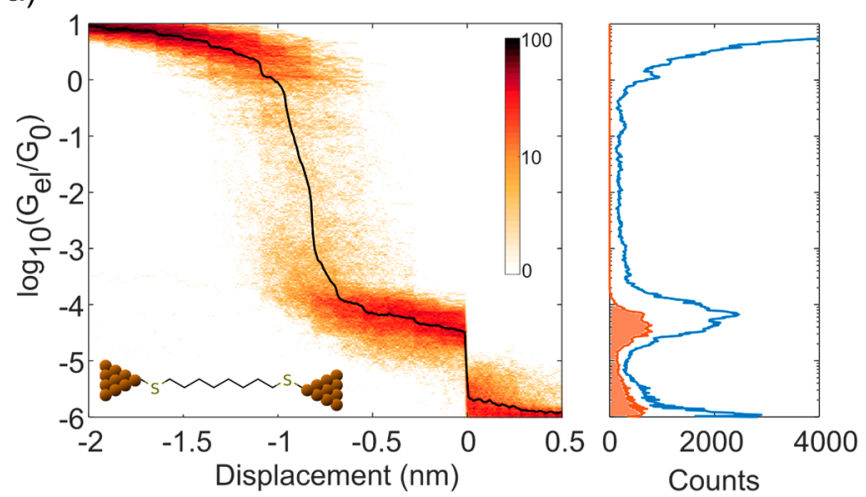

c)

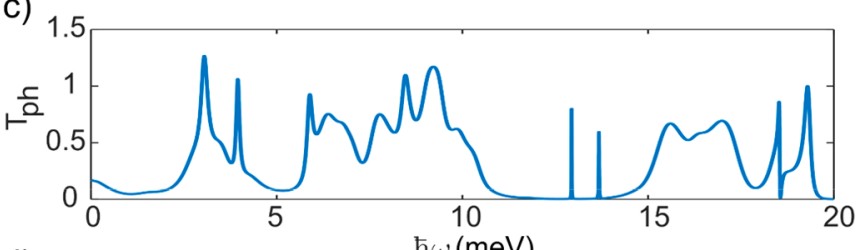

d)

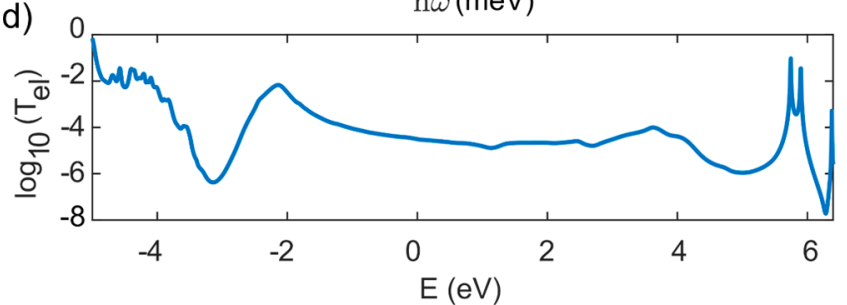

b)

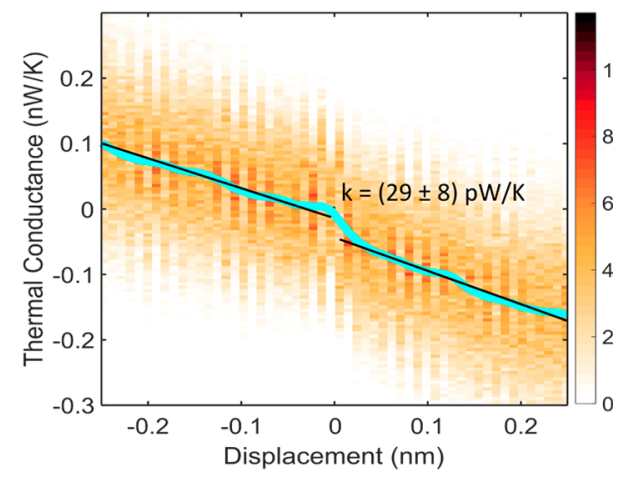

e)

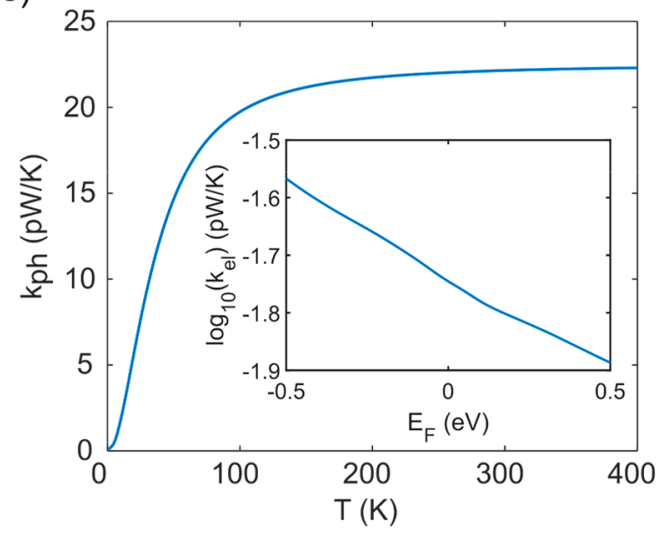

Figure 3. Experimental (a, b) and theoretical (c-e) results for octane-dithiol (ODT). (a) Electrical $2 \mathrm{D}$ and 1D histograms constructed with 615 traces measured at a fixed voltage of $90 \mathrm{mV}$ and a pulling speed $v=3 \mathrm{~nm} / \mathrm{s}$ at an angle of $40^{\circ}$ with respect to the MEMS surface. From the histograms, we can extract the most probable molecular electrical conductance of about $7 \times 10^{-5} G_{0}$. Note that the peak shifts to $5 \times 10^{-5} G_{0}$ before breaking (orange 1D histogram). (b) 2D Thermal conductance histogram. The width of the mean line (light blue) includes the total uncertainty of the extracted thermal conductance and shows a clear step around 0 (breaking point of the molecular junction), giving a thermal conductance $k=$ $(29 \pm 8) \mathrm{pW} / \mathrm{K}$. (c) Transmission coefficient $T_{\mathrm{ph}}$ of phonons with energy $\hbar \omega$. (d) Transmission coefficient $T_{\text {el }}$ of electrons with energy $E$. (e) Calculated thermal conductance $k_{\mathrm{ph}}$ due to phonons versus temperature $T$. The inset shows the thermal conductance due to electrons versus the Fermi energy at room temperature.

solvent exposure steps. Third, also the target molecules deposited from the solution that are not directly bridging the electrodes form a thermal contact. Such adsorbates, sketched as the green layer in Figure 1, are only weakly bonded to the gold electrodes and make a negligible contribution to the electrical conductance of the junction. They will be not chemically bound to both surfaces, but they can carry significant heat via the van der Waals bonds; ${ }^{5,12,14}$ they form a strong mechanical contact between tip and MEMS, with a typical thermal conductance of about $10-30 \mathrm{nW} / \mathrm{K}$ that depends on their relative distance. The reproducibility and the slope of the background signal are more important than its magnitude. We note that even in nominally clean experiments, ${ }^{15,16,27,28}$ in the near-field radiation regime exhibit background slopes between 0.5 and $8 \mathrm{nW} / \mathrm{K} / \mathrm{nm}$, similar to the range reported here.

Upon retracting the tip, the contact area with the adsorbate layer and the influence of radiation are gradually reduced, giving rise to the thermal background observed in the opening traces. Notably, thermal conductance variations versus tipsurface distance with similar slopes were recently measured in near-field heat transport measurements between nominally clean surfaces in UHV, even after thorough in situ cleaning procedures. This indicates that the contribution of radiation to the distance-dependent thermal conductance at $\mathrm{nm}$ distances cannot be excluded and most likely cannot be avoided experimentally. ${ }^{27,28}$ Under our experimental conditions, the thermal background is stable and does not significantly change during repeated opening and closing of the break-junction contact, remaining approximately linear in the region of analysis described below.

Figure 2 illustrates the experimental procedure to extract the thermal conductance of the OPE 3 molecule, using the electrical signal to verify the formation of molecular junctions. As in a standard break junction measurement, we collect typically 2000-5000 traces per data set and construct 1D and 2D histograms for the electrical (Figure 2a) and thermal (Figure $2 \mathrm{~b}$ ) conductance opening traces. We then rescale the displacement and the thermal conductance axis by shifting their origin to the breaking point of the molecular junction. This means that for every trace that has a defined molecular signature in the electrical signal (see section data analysis procedure of the Supporting Information), we locate the breaking point of the molecular plateau in the electrical conductance trace and use this to rescale the distance and thermal conductance traces. Rescaling here refers only to shifting the origin of the displacement axis to the breaking point of the molecular plateau signal in each curve (no stretching factor was introduced). In the literature, ${ }^{21,24,29}$ such shifts are made with respect to the conductance signal falling 
a)

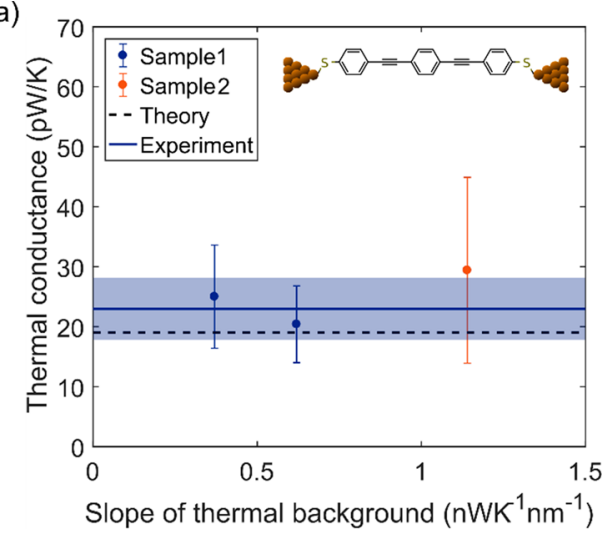

b)

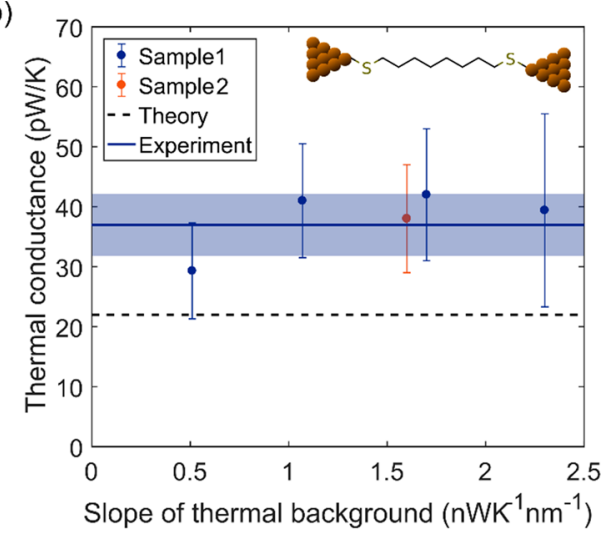

Figure 4. Summary of the experimental results obtained for OPE3 (a) and ODT (b). By performing the weighted average of the different experimental data points for each molecule, we obtain $k=(23 \pm 5) \mathrm{pW} / \mathrm{K}$ for OPE3 and $k=(37 \pm 5) \mathrm{pW} / \mathrm{K}$ for ODT. The experimental uncertainty is represented by the shaded region around the mean value (blue line). $1 \mathrm{D}$ and $2 \mathrm{D}$ histograms relative to the data points in panels a and $\mathrm{b}$ are provided in the Supporting Information.

below the conductance quantum, i.e., the breaking of the metal-metal contact. In any case, the rescaling helps to accommodate for drift and statistical variations of the breaking process.

Few examples of single traces with and without defined molecular plateaus are given in the Supporting Information. In this way, we can construct $2 \mathrm{D}$ histograms of the thermal conductance versus the displacement and average over the changes occurring in every trace when the junction breaks. To extract the thermal conductance of the molecule, we take the difference of the linear fits of the mean of the thermal background before and after the breaking point, see Figure $2 \mathrm{~b}$. The variation of the slopes extracted from the fitting of different data sets appears random and is included in the reported uncertainty. On the small fitting range of few Ångströms, nonlinear fits did not yield better results. On a larger length scale, however, there is no prior assumption on the linearity of the background signal.

Applying this method to dithiol-OPE3s, Figure 2a,b, we first note that the observed single peak in the electrical conductance histogram at $2.7 \times 10^{-4} G_{0}$ (where $G_{0}=2 \mathrm{e}^{2} / h$ is the electrical conductance quantum), is in excellent agreement with several independent reports in different environmental conditions ${ }^{17,29,30}$ indicating single-molecule OPE3 junctions. The mean of the $2 \mathrm{D}$ thermal histogram shows a clear step in the thermal background at the breaking point of the molecular junction $(d=0 \mathrm{~nm})$, Figure $2 \mathrm{~b}$. The difference at 0 between the linear fits of the mean gives a molecular thermal conductance $k=(20 \pm 6) \mathrm{pW} / \mathrm{K}$.

To construct a theoretical description of thermal transport, we use density functional theory (DFT) to obtain the optimized geometry of each junction. We then use the harmonic approximation to calculate forces on each atom in order to study the vibrational properties of the junctions. Using these force matrices, we obtain a dynamical matrix and combine it with the Green's function method to calculate the transmission coefficient $T_{\mathrm{ph}}(\hbar \omega)$ of phase-coherent phonons with energy $\hbar \omega$ traversing from one electrode to the other. The thermal conductance due to phonons is then obtained from $T_{\mathrm{ph}}(\hbar \omega)$ using a Landauer-like formula ${ }^{8,31}$ (see computational methods in the Supporting Information). Figure 2C shows the phonon transmission coefficient $T_{\mathrm{ph}}$ of OPE3. Due to the low Debye frequency of gold electrodes $(\sim 20 \mathrm{meV}){ }^{8}$ phonons with energies higher than $20 \mathrm{meV}$ are filtered (Figure 2c) and the phonon thermal conductance for temperatures higher than $\sim 200 \mathrm{~K}$ (Figure $2 \mathrm{e}$ ) saturates to $19 \mathrm{pW} / \mathrm{K}$ in excellent agreement with the experimental data.

In order to calculate the electrical properties of the junctions, we combine DFT mean-field Hamiltonians of each junction with the Green's function method to calculate transmission coefficient $T_{\mathrm{el}}(E)$ for electrons traversing from the hot electrode to the cold one (Figure 2d) through the molecules (see computational methods in the Supporting Information). The electron transmission through OPE3 is dominated by their highest occupied molecular orbitals (HOMO) in agreement with previous reports. ${ }^{18}$ The electrical conductance is then obtained using the Landauer formula. At low temperatures, the conductance $G=G_{0} T_{\mathrm{el}}\left(E_{\mathrm{F}}\right)$ where $E_{\mathrm{F}}$ is the Fermi energy of electrodes. ${ }^{31}$ In addition, the room temperature thermal conductance due to electrons can be calculated from the electron transmission $T_{\mathrm{el}}$ as a function of the electrodes Fermi energy $E_{\mathrm{F}}$, as shown in the inset of Figure 2e. At the DFT predicted Fermi energy $\left(E_{\mathrm{F}}=0\right)$, the thermal conductance due to electrons is $<0.1 \mathrm{pW} / \mathrm{K}$, which is much smaller than the phonon contribution. In addition, for a wide energy range around $E_{\mathrm{F}}=0$, the contribution of electrons to the total thermal conductance is negligible.

The same experimental and theoretical methods were then applied to study the thermal transport properties of ODT (Figure 3). In contrast to OPE3, the junction dynamics of ODT show stronger variation in independent reports. Different conductance values observed are attributed to various binding configurations of the S-Au bond, ${ }^{23,32}$ gauche defects, ${ }^{24,33}$ and a higher likeliness to observe multiple-molecules in a single junction. ${ }^{21,34}$ Nevertheless, there is some consensus about the frequently observed electrical conductance of $5 \times 10^{-5} G_{0}$ relating to the atop-atop configuration of a single stretched ODT the molecule. ${ }^{21}$ Most of the histograms show a prominent peak at $5-7 \times 10^{-5} G_{0}$, which we attribute to the atop-atop configuration. With lower probability, we observe plateaus at $1 \times 10^{-5} G_{0}$ and at about $1 \times 10^{-4} G_{0}$, in agreement with a recent robust statistical approach. ${ }^{24}$ Therefore, to simplify the analysis of the thermal signal, we focus on the atop-atop junction configuration considering molecular traces with plateaus between 2 and $8 \times 10^{-5} G_{0}$. For this junction geometry, we obtain a thermal conductance $k=(29 \pm 8) \mathrm{pW} /$ 
K. Simulations were based on the atop-atop configuration (inset of Figure 3a) resulting in a thermal conductance value of $22 \mathrm{pW} / \mathrm{K}$ at room temperature (Figure $3 \mathrm{e}$ ) in reasonable agreement.

When the tip is pulled from the electrode surface even with an angle, there may be a backlash because the system has a limited mechanical compliance. ${ }^{15}$ This backlash can translate into a modification of the thermal signal due to the distancedependent thermal background. In what follows, our aim is to demonstrate that the effect of a backlash is not significant in our case.

First, for a given stiffness of the MEMS and the stiffness of a molecular bond, the backlash should lead to a certain backlash distance. Then, for different slopes of the thermal background signal, we should have an apparent step in the thermal conductance trace in proportion to the slope of the background signal. To test this, we repeated the experiment for both OPE3 and ODT on the same respective sample on different spots and on different samples (Figure 4). We found the slope of the background varying from measurement to measurement within certain limits, while the MEMS stiffness should be approximately constant. Within our uncertainty, we do not observe any significant dependence of the thermal conductance of OPE3 and ODT on the background slope (Figure 4). Therefore, we can argue that the backlash cannot be significant. By taking the weighted average of the different data points, we obtain $k=(23 \pm 5) \mathrm{pW} / \mathrm{K}$ for OPE3 and $k=$ $(37 \pm 5) \mathrm{pW} / \mathrm{K}$ for ODT. We note, the same method was applied to gold-gold junctions leading to the results reported by two independent studies, ${ }^{15,16}$ Figure S3. In particular, we obtain a thermal conductance of $540 \mathrm{pW} / \mathrm{K}$ for a single gold atom contact (or atomic chain), which is very close to the value predicted by the Wiedemann-Franz law of $577 \mathrm{pW} / \mathrm{K}$, for one electrical conductance quantum at $T=305 \mathrm{~K}$.

A second independent argument that a backlash cannot be responsible for the observed step-change in thermal conductance comes from performing a test using closing traces. The junction formation yield is typically smaller for closing traces. Nevertheless, we were able to collect sufficient traces in the case of ODT with a clear molecular signature. We obtained similar values of thermal conductance upon forming molecular junctions within closing traces $(k=35 \pm 7 \mathrm{pW} / \mathrm{K}$ from 440 traces, see Figure S4). The mechanics of a loaded spring is very different for a closing trace, in which a junction can be formed with a much reduced or even insignificant backlash. We estimate that a sensor stiffness of $>20 \mathrm{~N} / \mathrm{m}$ is required to produce this result (Supporting Information).

Finally, it is well-known that bond breaking is a thermally activated process that can be accelerated exponentially by an applied pulling force. Spontaneous, and therefore backlashfree, bond breaking is favored if sufficient time is given. Huang et al. ${ }^{35}$ reported spontaneous bond breaking of ODT junctions at pulling velocities below $7 \mathrm{~nm} / \mathrm{s}$ using an STM tip. Nef et al. $^{36}$ observed only a minority of breaking processes accompanied by measurable backlash using a sensor of 3-4 $\mathrm{N} / \mathrm{m}$ pulling at $15 \mathrm{~nm} / \mathrm{s}$. Increasing the pulling velocity to 18 and $40 \mathrm{~nm} / \mathrm{s}$ (with stiffer sensors) finally resulted in appreciable backlash according to other reports. ${ }^{37,38}$ In contrast, our experiments use the pulling speed of $2-3 \mathrm{~nm} /$ s. Therefore, we expect no backlash, taking into account these literature items.

A further contributing effect to the absence of a backlash is that the nonchemically bound molecules were contributing to the thermal background, which also stabilize the junction mechanically. This, when combined with the contribution of the in-plane stiffness of the MEMS, can lead to an effective stiffness larger than the chemical bonds of the molecular junction. Evidence for this is the fact that we can controllably break a gradually thinning metal-metal contact. The stiffness of an initial multi atom-wide contact is much larger than a single molecular bond. Therefore, either the restoring force reduces significantly with pulling distance, or, otherwise, the contact would directly rupture from a larger contact area instead of going through the gradual changes. In fact, the latter is what we observe, if we perform experiments at a vertical pulling angle with only the smaller normal stiffness of the MEMS.

One confirmation that either the spring constant must be larger than $\sim 10 \mathrm{~N} / \mathrm{m}$ or the breaking force must be smaller than $0.1 \mathrm{nN}$ can also be concluded from the fact that the $\mathrm{Au}-$ $\mathrm{Au}$ contact can be gradually broken. As seen in the individual sample traces, breaking from a $2 G_{0}$ or $3 G_{0}$ plateau to a $1 G_{0}$ plateau can be observed in many traces. If the break force was in the force-driven regime, then the tip would apply a force of $2-3 \mathrm{nN}$ to the $2-3 \mathrm{G}_{0}$ contact. The backlash after breaking of this would have to be efficient enough to reduce the force to below $1 \mathrm{nN}$ as to not immediately break the $1 G_{0}$ bond during the same breaking event. However, the maximum backlash would be the length of stretching of a single atom gold wire, and at room temperature, the average plateau length is below $0.1 \mathrm{~nm}$ (Supporting Information). In contrast, a spring constant of only $1 \mathrm{~N} / \mathrm{m}$ combined with a breaking force of 1 $\mathrm{nN}$ needs a minimum of $2 \mathrm{~nm}$ to relieve a force related to the transition from $3 G_{0}$ to $1 G_{0}$.

The Supporting Information comprises a detailed discussion on the potential error induced by the backlash issues based on the above arguments.

From studies of thermal transport across self-assembled monolayers, ${ }^{41}$ we learn that the environment of a molecule can influence its vibrational freedom and therefore the transport characteristic. In break junction experiments, however, the molecule is not in a densely packed configuration such as in self-assembled monolayers. Therefore, the environment has no significant influence on the thermal conductance other than providing a slowly varying background. The expected mobility of unbound molecules and the immobility of molecules bound to one of the electrodes leads us to believe that molecular rearrangement cannot be a source of major influence on the observed step change. The relatively slow measurement (compared to the molecular rearrangements time scale) and statistical analysis is expected to average out different configurations of surrounding molecules and the probed junction.

We not only find good agreement between theory and experimental data but also note that our data agrees with expected bounds from reference data on similar systems. While heat transport of the thiol-bound OPE3 system has not been studied before, there are simulations (based on a similar approach to ours) for Au-OPE3-Au junction using amine anchor groups ${ }^{2}$ yielding $\sim 24 \mathrm{pW} / \mathrm{K}$, suggesting a very similar bonding strength of the respective anchor groups. Simulations of OPE3 bound to silicon ${ }^{3}$ predicting $\sim 60 \mathrm{pW} / \mathrm{K}$ highlights the role of a higher Debye temperature of $\mathrm{Si}$ compared to $\mathrm{Au}$ electrodes, which allows higher frequency phonon transport leading to higher thermal conductance. 
Thermal transport through SAMs of alkanethiols between different materials, in contrast, have been studied using different methods, demonstrating that the thermal conductance is independent of the molecular length with more than 8 C atoms. ${ }^{12,39,40}$ Interesting phonon filtering effects were instead simulated and probed for shorter chains. ${ }^{14}$ Thermal conductance values up to a maximum of $14 \mathrm{pW} / \mathrm{K}$ were reported for $\mathrm{C}_{10} \mathrm{~S}_{2}$ and $\mathrm{C}_{11} \mathrm{~S}_{2}$ with gold electrodes, ${ }^{13,41}$ in contrast with the usually higher values predicted by molecular dynamics (MD) simulations ranging between $25 \mathrm{pW} / \mathrm{K}^{11,13}$ and $45 \mathrm{pW} / \mathrm{K}^{42,43}$ This discrepancy is attributed to uncertainty in the number of molecules in contact with the electrodes because of roughness. Interestingly, our experimental and theoretical results fit well with the available MD simulations. Another ab initio study ${ }^{44}$ predicts $k=35 \mathrm{pW} / \mathrm{K}$ for ODT between gold electrodes. We attribute this variation in the predictions and experimental results to the complex behavior of the molecule in the junction, which includes not only bonding geometries but also the ability of the ODT to form gauche defects. This needs further systematic investigations in future studies.

Thermal transport in both OPE3 and ODT is dominated by phonons with negligible contributions from electronic transport channels. The electrical conductance of OPE3 is higher than that of ODT for a wide range of energies around the DFT Fermi energy $\left(E_{\mathrm{F}}=0\right)$, as shown in Figures $2 \mathrm{~d}$ and $3 \mathrm{~d}$. Consequently, the room temperature thermal conductance of ODT due to electrons is lower than that of OPE3 for a wide range of Fermi energies, in agreement with the WiedemannFranz law (inset of Figures 2 and $3 \mathrm{e}$ ). At the DFT Fermi energy $E_{\mathrm{F}}=0$, the electronic thermal conductance of OPE3 and ODT are $0.07 \mathrm{pW} / \mathrm{K}$ and $0.01 \mathrm{pW} / \mathrm{K}$, respectively. Although the electronic contribution to heat transport is found negligible in these systems, it is interesting to discuss, why a deviation from the Wiedemann-Franz law could lead to a more appreciable contribution. The Wiedemann-Franz law holds only when electron transmission coefficient $T(E)$ is linear in the scale of $k T$. In both OPE3 and ODT, $T(E)$ is linear in the scale of $k T(25 \mathrm{meV}$ at room temperature $)$ in the vicinity of Fermi energy (Figures $2 \mathrm{~d}$ and $3 \mathrm{~d}$ ). Therefore, the Wiedemann-Franz law holds, and $\kappa_{\mathrm{el}}=\alpha T G$, where $\alpha$ is the Lorenz number, $T$ is temperature, and $G$ is electrical conductance

It is worth to mention that we have chosen two molecular backbones, which by chemical design are different. The OPE3 molecule has double and triple bonds and is very stiff compared to the more compliant and flexible alkane backbone. A stiffer material has in general higher phonon propagation speed (or velocities of sound) leading to larger thermal conductance. However, we observe that ODT is the better thermal conductor. This can be explained within the phonon mismatch picture. The frequencies of heat-carrying phonons in the gold electrodes are relatively low due to the large mass of the gold nuclei. To quantify, we have to remember that a sum over all phonon frequencies needs to be made to account for all heat transport, which reaches $20 \mathrm{meV}$. The phonon transmission spectra of Figures $2 c$ (OPE3) and $3 c$ (ODT) show transmitting modes in this range. The videos in the Supporting Information show the motion of both OPE3 and ODT due to different phononic modes below the Debye frequency of gold. While modes are found in both, the width of the phonon transmission resonances overall is greater in ODT (Figure 3c) compared with OPE3 (Figure 2c), which implies a higher density of phonon states at the connection point to electrodes in ODT.

Nevertheless, the difference in thermal conductance between the two systems is less than a factor of 2 in the experiments. This underpins the more general notion that the variation in thermal conductivity between solid materials is rather limited when compared to the tunability of charge or photon transport in matter. In order to manipulate thermal transport, we, therefore, need to employ strategies based on phonon engineering in molecular systems carefully. These include, for example, placing Fano-resonances in the integration window of frequencies offered by the electrodes to reduce thermal conduction. ${ }^{9,10}$ Furthermore, phonon interference of two conduction paths along a molecule is another interesting candidate mechanism. The proposed mechanisms, in general, rely on a specific transport regime. One of the main aspects of the theoretical description allied here is the phase coherence nature of transport. Through the quantitative agreement between experiment and theory, one of our main results is the confirmation of the phonon transport being phasecoherent along these junctions.

In conclusion, we demonstrated the first measurement of the thermal conductance of single-molecule junctions. We applied this method to the two benchmark molecules OPE3 and ODT and found good agreement with our ab initio simulations, based on phase-coherent heat transport at the single-molecule level. Given the versatility of the approach, we expect this work to enable systematic investigations of molecular-scale heat transport, opening new possibilities for engineering materials with tailored thermal transport properties.

\section{ASSOCIATED CONTENT}

\section{Supporting Information}

The Supporting Information is available free of charge on the ACS Publications website at DOI: 10.1021/acs.nanolett.9b02089.

Computational and experimental methods, experimental data analysis procedure additional DFT calculations, thermal conductance, electrical and thermal conductance histograms, and experimental results (PDF)

Video S1 (MP4)

Video S2 (MP4)

\section{AUTHOR INFORMATION}

\section{Corresponding Authors}

*E-mail: Hatef.Sadeghi@warwick.ac.uk.

*E-mail: bgo@zurich.ibm.com.

ORCID $\odot$

Hatef Sadeghi: 0000-0001-5398-8620

Colin Lambert: 0000-0003-2332-9610

Bernd Gotsmann: 0000-0001-8978-7468

\section{Notes}

The authors declare no competing financial interest.

The raw STM-BJ data can be found in the IBM repository available at: https://zenodo.org/badge/DOI/10.5281/zenodo. 3521318.svg. The input files to reproduce simulation data can be found at: https://warwick.ac.uk/fac/sci/wcpm/people/ hsadeghi/repository. 


\section{ACKNOWLEDGMENTS}

The authors gratefully acknowledge management support from K. Moselund, W. Riess, and J. Repp and technical support from S. Karg, M. Tschudy, H. Wolf, E. Lörtscher, S. Reidt, A. Olziersky, D. Dávila Pineda, F. Koenemann, P. Nirmalraj, A. Prasmusinto, and F. Menges. Special thanks go to K. Weiland and M. Mayor for the purification step of the OPE3 molecules. The authors acknowledge the Leverhulme Trust for Leverhulme Early Career Fellowships nos. ECF-2017-186 and ECF-2018-375 and UKRI for Future Leaders Fellowship no. MR/S015329/1. Further support from the U.K. EPSRC is acknowledged, through grant nos. EP/M014452/1, EP/ P027156/1, EP/N017188/1, and EP/N03337X/1. This project has received funding from the European Union's FP7 ITN "MOLESCO" project no. 606728 and Horizon 2020 research and innovation programs under grant agreement nos. 767187 (QuIET) and 766853 (EFINED).

\section{REFERENCES}

(1) Hu, L.; Zhang, L.; Hu, M.; Wang, J. S.; Li, B.; Keblinski, P. Phonon Interference at Self-Assembled Monolayer Interfaces: Molecular Dynamics Simulations. Phys. Rev. B: Condens. Matter Mater. Phys. 2010, 81 (23), 1-5.

(2) Martin, C. A.; Smit, R. H. M.; Egmond, R. Van; Van Der Zant, H. S. J.; Van Ruitenbeek, J. M. A Versatile Low-Temperature Setup for the Electrical Characterization of Single-Molecule Junctions. Rev. Sci. Instrum. 2011, 82 (5), 053907.

(3) Markussen, T. Phonon Interference Effects in Molecular Junctions. J. Chem. Phys. 2013, 139 (24), 244101.

(4) Segal, D.; Nitzan, A. Heat Rectification in Molecular Junctions. J. Chem. Phys. 2005, 122 (19), 194704.

(5) Segal, D.; Nitzan, A.; Hänggi, P. Thermal Conductance through Molecular Wires. J. Chem. Phys. 2003, 119 (13), 6840-6855.

(6) Segal, D.; Agarwalla, B. K. Vibrational Heat Transport in Molecular Junctions. Annu. Rev. Phys. Chem. 2016, 67 (1), 185-209.

(7) Cuevas, J. C.; Scheer, E. World Scientific 2017, 15, 1.

(8) Sadeghi, H.; Sangtarash, S.; Lambert, C. J. Oligoyne Molecular Junctions for Efficient Room Temperature Thermoelectric Power Generation. Nano Lett. 2015, 15 (11), 7467-7472.

(9) Famili, M.; Grace, I.; Sadeghi, H.; Lambert, C. J. Suppression of Phonon Transport in Molecular Christmas Trees. ChemPhysChem 2017, 18 (10), 1234-1241.

(10) Sadeghi, H. Quantum and Phonon Interference-Enhanced Molecular-Scale Thermoelectricity. J. Phys. Chem. C 2019, 123 (20), 12556-12562.

(11) Duda, J. C.; Saltonstall, C. B.; Norris, P. M.; Hopkins, P. E. Assessment and Prediction of Thermal Transport at Solid-SelfAssembled Monolayer Junctions. J. Chem. Phys. 2011, 134 (9), No. 094704.

(12) Losego, M. D.; Grady, M. E.; Sottos, N. R.; Cahill, D. G.; Braun, P. V. Effects of Chemical Bonding on Heat Transport across Interfaces. Nat. Mater. 2012, 11 (6), 502-506.

(13) Majumdar, S.; Sierra-Suarez, J. A.; Schiffres, S. N.; Ong, W. L.; Higgs, C. F.; McGaughey, A. J. H.; Malen, J. A. Vibrational Mismatch of Metal Leads Controls Thermal Conductance of Self-Assembled Monolayer Junctions. Nano Lett. 2015, 15 (5), 2985-2991.

(14) Meier, T.; Menges, F.; Nirmalraj, P.; Hölscher, H.; Riel, H.; Gotsmann, B. Length-Dependent Thermal Transport along Molecular Chains. Phys. Rev. Lett. 2014, 113 (6), No. 060801.

(15) Mosso, N.; Drechsler, U.; Menges, F.; Nirmalraj, P.; Karg, S.; Riel, H.; Gotsmann, B. Heat Transport through Atomic Contacts. Nat. Nanotechnol. 2017, 12 (5), 430-433.

(16) Cui, L.; Jeong, W.; Hur, S.; Matt, M.; Klöckner, J. C.; Pauly, F.; Nielaba, P.; Cuevas, J. C.; Meyhofer, E.; Reddy, P. Quantized Thermal Transport in Single-Atom Junctions. Science (Washington, DC, U. S.) 2017, 355 (6330), 1192-1195.
(17) Wu, S.; González, M. T.; Huber, R.; Grunder, S.; Mayor, M.; Schoenenberger, C.; Calame, M. Molecular Junctions Based on Aromatic Coupling. Nat. Nanotechnol. 2008, 3 (9), 569-574.

(18) Frisenda, R.; Tarkuç, S.; Galán, E.; Perrin, M. L.; Eelkema, R.; Grozema, F. C.; van der Zant, H. S. J. Electrical Properties and Mechanical Stability of Anchoring Groups for Single-Molecule Electronics. Beilstein J. Nanotechnol. 2015, 6 (1), 1558-1567.

(19) Gonzalez, M. T.; Leary, E.; García, R.; Verma, P.; Herranz, M. Á.; Rubio-Bollinger, G.; Martín, N.; Agrait, N. Break-Junction Experiments on Acetyl-Protected Conjugated Dithiols under Different Environmental Conditions. J. Phys. Chem. C 2011, 115 (36), 1797317978.

(20) Kaliginedi, V.; Moreno-Garcia, P.; Valkenier, H.; Hong, W.; García-Suárez, V. M.; Buiter, P.; Otten, J. L. H.; Hummelen, J. C.; Lambert, C. J.; Wandlowski, T. Correlations between Molecular Structure and Single Junction Conductance: A Case Study with OPEType Wires. J. Am. Chem. Soc. 2012, 134, 5262-5275.

(21) Gonza, M. T.; Wu, S.; Huber, R.; Molen, S. J. Van Der; Schönenberger, C.; Calame, M. Electrical Conductance of Molecular Junctions by a Robust Statistical Analysis. Nano Lett. 2006, 6 (10), $2238-2242$

(22) Jang, S. Y.; Reddy, P.; Majumdar, A.; Segalman, R. A. Interpretation of Stochastic Events in Single Molecule Conductance Measurements. Nano Lett. 2006, 6 (10), 2362-2367.

(23) Li, C.; Pobelov, I.; Wandlowski, T.; Bagrets, A.; Arnold, A.; Evers, F. Charge Transport in Single Au I Alkanedithiol I Au Junctions: Coordination Geometries and Conformational Degrees of Freedom. J. Am. Chem. Soc. 2008, 130 (1), 318-326.

(24) Lemmer, M.; Inkpen, M. S.; Kornysheva, K.; Long, N. J.; Albrecht, T. Unsupervised Vector-Based Classification of SingleMolecule Charge Transport Data. Nat. Commun. 2016, 7, 12922.

(25) Lörtscher, E.; Widmer, D.; Gotsmann, B. Next-Generation Nanotechnology Laboratories with Simultaneous Reduction of All Relevant Disturbances. Nanoscale 2013, 5, 10542.

(26) Huang, C.; Rudnev, A. V.; Hong, W.; Wandlowski, T. Break Junction under Electrochemical Gating: Testbed for Single-Molecule Electronics. Chem. Soc. Rev. 2015, 44 (4), 889-901.

(27) Cui, L.; Jeong, W.; Fernández-Hurtado, V.; Feist, J.; GarcíaVidal, F. J.; Cuevas, J. C.; Meyhofer, E.; Reddy, P. Study of Radiative Heat Transfer in Ångström- and Nanometre-Sized Gaps. Nat. Commun. 2017, 8, 1.

(28) Kloppstech, K.; Könne, N.; Biehs, S. A.; Rodriguez, A. W.; Worbes, L.; Hellmann, D.; Kittel, A. Giant Heat Transfer in the Crossover Regime between Conduction and Radiation. Nat. Commun. 2017, 1 .

(29) Frisenda, R.; Janssen, V. A. E. C.; Grozema, F. C.; Van Der Zant, H. S. J.; Renaud, N. Mechanically Controlled Quantum Interference in Individual Ï-Stacked Dimers. Nat. Chem. 2016, 8 (12), 1099-1104.

(30) Lissau, H.; Frisenda, R.; Olsen, S. T.; Jevric, M.; Parker, C. R.; Kadziola, A.; Hansen, T.; Van Der Zant, H. S. J.; Brøndsted Nielsen, M.; Mikkelsen, K. V. Tracking Molecular Resonance Forms of DonorAcceptor Push-Pull Molecules by Single-Molecule Conductance Experiments. Nat. Commun. 2015, 6, 1-8.

(31) Sadeghi, H. Theory of Electron, Phonon and Spin Transport in Nanoscale Quantum Devices. Nanotechnology 2018, 29 (37), 373001.

(32) Haiss, W.; Martín, S.; Leary, E.; Van Zalinge, H.; Higgins, S. J.; Bouffier, L.; Nichols, R. J. Impact of Junction Formation Method and Surface Roughness on Single Molecule Conductance. J. Phys. Chem. C 2009, 113, 5823.

(33) Suzuki, M.; Fujii, S.; Fujihira, M. Measurements of Currents through Single Molecules of Alkanedithiols by Repeated Formation of Break Junction in Scanning Tunneling Microscopy under Ultrahigh Vacuum. Japanese J. Appl. Physics, Part 1 Regul. Pap. Short Notes Rev. Pap. 2006, 45, 2041.

(34) Li, X.; He, J.; Hihath, J.; Xu, B.; Lindsay, S. M.; Tao, N. Conductance of Single Alkanedithiols: Conduction Mechanism and Effect of Molecule-Electrode Contacts. J. Am. Chem. Soc. 2006, 128 (6), 2135-2141. 
(35) Huang, Z.; Chen, F.; Bennett, P. A.; Tao, N. Single Molecule Junctions Formed via Au-Thiol Contact: Stability and Breakdown Mechanism. J. Am. Chem. Soc. 2007, 129 (43), 13225-13231.

(36) Nef, C.; Frederix, P. L. T. M.; Brunner, J.; Schönenberger, C.; Calame, M. Force-Conductance Correlation in Individual Molecular Junctions. Nanotechnology 2012, 23, 365201.

(37) Frei, M.; Aradhya, S. V.; Hybertsen, M. S.; Venkataraman, L. Linker Dependent Bond Rupture Force Measurements in SingleMolecule Junctions. J. Am. Chem. Soc. 2012, 134, 4003.

(38) Xu, B.; Xiao, X.; Tao, N. J. Measurements of Single-Molecule Electromechanical Properties. J. Am. Chem. Soc. 2003, 125, 16164.

(39) Wang, Z.; Carter, J. A.; Lagutchev, A.; Koh, Y. K.; Seong, N. H.; Cahill, D. G.; Dlott, D. D. Ultrafast Flash Thermal Conductance of Molecular Chains. Science (Washington, DC, U. S.) 2007, 317 (5839), 787-790.

(40) Wang, R. Y.; Segalman, R. A.; Majumdar, A. Room Temperature Thermal Conductance of Alkanedithiol Self-Assembled Monolayers. Appl. Phys. Lett. 2006, 89 (17), 27-30.

(41) Majumdar, S.; Malen, J. A.; McGaughey, A. J. H. H. Cooperative Molecular Behavior Enhances the Thermal Conductance of Binary Self-Assembled Monolayer Junctions. Nano Lett. 2017, 17 (1), 220-227.

(42) Luo, T.; Lloyd, J. R. Non-Equilibrium Molecular Dynamics Study of Thermal Energy Transport in Au-SAM-Au Junctions. Int. J. Heat Mass Transfer 2010, 53, 1.

(43) Luo, T.; Lloyd, J. R. Equilibrium Molecular Dynamics Study of Lattice Thermal Conductivity/Conductance of Au-SAM-Au Junctions. J. Heat Transfer 2009, 032401.

(44) Klockner, J. C.; Burkle, M.; Cuevas, J. C.; Pauly, F. Length Dependence of the Thermal Conductance of Alkane-Based SingleMolecule Junctions: An Ab Initio Study. Phys. Rev. B: Condens. Matter Mater. Phys. 2016, 94 (20), 1-9. 